RESEARCH PAPER RP1232

Part of Journal of Research of the National Bureau of Standards, Volume 23, August 1939

\title{
PARTICLE SIZE AND PLASTICITY OF LIME
}

\author{
By Dana L. Bishop
}

\section{ABSTRACT}

Particle-size distributions and Emley plasticity values were determined on 25 hydrated limes. The geometric weight-mean diameters varied from 2.9 to 7.8 microns. There was no relation between their particle-size distributions down to 2 microns and their Emley plasticity values. Hydrated limes having very similar particle-size distributions had widely different plasticities. Particle-size measurements down to about 1 micron were made on quicklime putties prepared by hydrating quicklimes with an excess of water. Putties prepared in such a manner were very much finer than the commercial hydrated limes. There was also no relation between the distributions down to 1 micron and the Emley plasticity values of the quicklime putties. Limes having the same calculated specific surface may have widely different particle-size distributions. It is possible that the size distribution of the material finer than 2 microns may be the determining factor in the plasticity of limes.

\section{CONTENTS}

Page

I. Introduction $\ldots \ldots \ldots \ldots$

II. Measurements _...

1. Emley plasticity values

2. Particle size

III. Particle-size distributions

IV. Particle size and Emley plasticity values of hydrates $\ldots \ldots \ldots$

V. Particle size and Emley plasticity values of quicklime putties $\ldots \ldots \ldots$

VI. Summary _............ 292

\section{INTRODUCTION}

Very few data are available concerning the particle-size distribution of commercial hydrated limes, especially for that portion which is finer than 10 microns. For this reason and because of the recent interest in the plasticity of lime, these data relating to the particle size and Emley plasticity values of hydrated limes and quicklime putties are presented.

\section{MEASUREMENTS}

\section{EMLEY PLASTICITY VALUES}

Plasticity measurements of the hydrated limes and quicklime putties were made with the Emley plasticimeter, the instrument commonly used in the lime industry to measure plasticity. ${ }^{1}$ The limes were

1 SS-L-351, Federal Specification for Lime; Hydrated (for) Structural Purposes. Also Standard Specifications for Hydrated Lime for Structural Purposes, ASTM Designation C-6-31, Am. Soc. for Testirg Materials, Standards, pt. 2, Non-Metals, p. 30 (1936). 
made into stiff putties with water and allowed to soak overnight, after which they were brought to standard consistency by the gradual addition of water accompanied by vigorous stirring, thus obtaining maximum plasticity.

\section{PARTICLE SIZE}

The particle-size measurements reported in this paper were made by a method previously described. ${ }^{2}$ This method consists essentially in weighing the lime as it settles out of suspension in a liquid, and calculating the particle-size distribution from the sedimentation record. Anhydrous butanol was used as a dispersion medium. This medium was chosen instead of water because it gives better dispersion of the lime. When lime is dispersed in water, agglomeration takes place, which alters the rate of sedimentation very much and would yield erroneous sedimentation data. In dilute suspensions in water much of the finer fraction is also lost through the interaction of the lime and carbon dioxide, with consequent solution and reduction in size of some of the particles. This seriously affects the particle-size indications, especially of the finer sizes. Sedimentation measurements were therefore made on dry hydrated lime after dispersing it in butanol with the aid of a stirring device.

\section{PARTICLE-SIZE DISTRIBUTION}

The sedimentation data of the hydrated limes indicated that many of them contained large amounts of very fine material. This fine material settled so slowly that it was impractical to obtain a complete sedimentation curve. Hence the sedimentations were ordinarily continued only sufficiently long to give points on the distribution curve down to about 2 microns for the hydrated limes and about 1 micron for quicklime putties.

In order to calculate any of the constants, such as mean diameter or surface area, which are used to describe a distribution, it was necessary to make some assumption regarding the distribution of the material below 2 microns in size. Hatch and Choate ${ }^{3}$ have shown that the particle-size distribution of certain materials is such that if on logarithmic-probability paper the particle diameters are plotted on the logarithmic scale and the cumulative percentages of the total number of particles on the probability scale, the points fall on a straight line. ${ }^{4}$ It can be shown that if a distribution is of this nature, the percentage by weight coarser than each size can be plotted instead of the number of particles, and these points will likewise fall on a straight line. The cumulative weight distribution of each of the 25 hydrated limes of this investigation was plotted on logarithmic-probability paper and in each case the points fell very close to a straight line (see fig. 1). It was therefore assumed in obtaining the geometric mean

\footnotetext{
${ }^{2}$ D. L. Bishop, BS J. Research 12, 175 (1934) RP642.

$3 \mathrm{~T}$. Hatch and S. P. Choate, Statistical description of particle size of nonuniform substance, J. Frank. Insı. 207, $369(1929)$.

$4 \mathrm{~A}$ straight line on such paper represents a distribution following the law
}

$$
y d(\ln x)=\frac{1}{\sqrt{2 \pi} \ln \lambda} e^{-\frac{\ln ^{2} x / g}{2 \ln ^{2} \lambda}} d(\ln x)
$$

that is, the logarithm of $x$ is distributed normally. 
diameters, the geometric standard deviation, ${ }^{5}$ and the specific surfaces that for each lime the diameter distribution of the particles smaller than 2 microns was represented by an extrapolation of the straight line fitted to the observed points for particles greater than 2 microns. Hatch and Choate have shown how for such a straight-line distribution the geometric mean and the standard geometric deviation can be

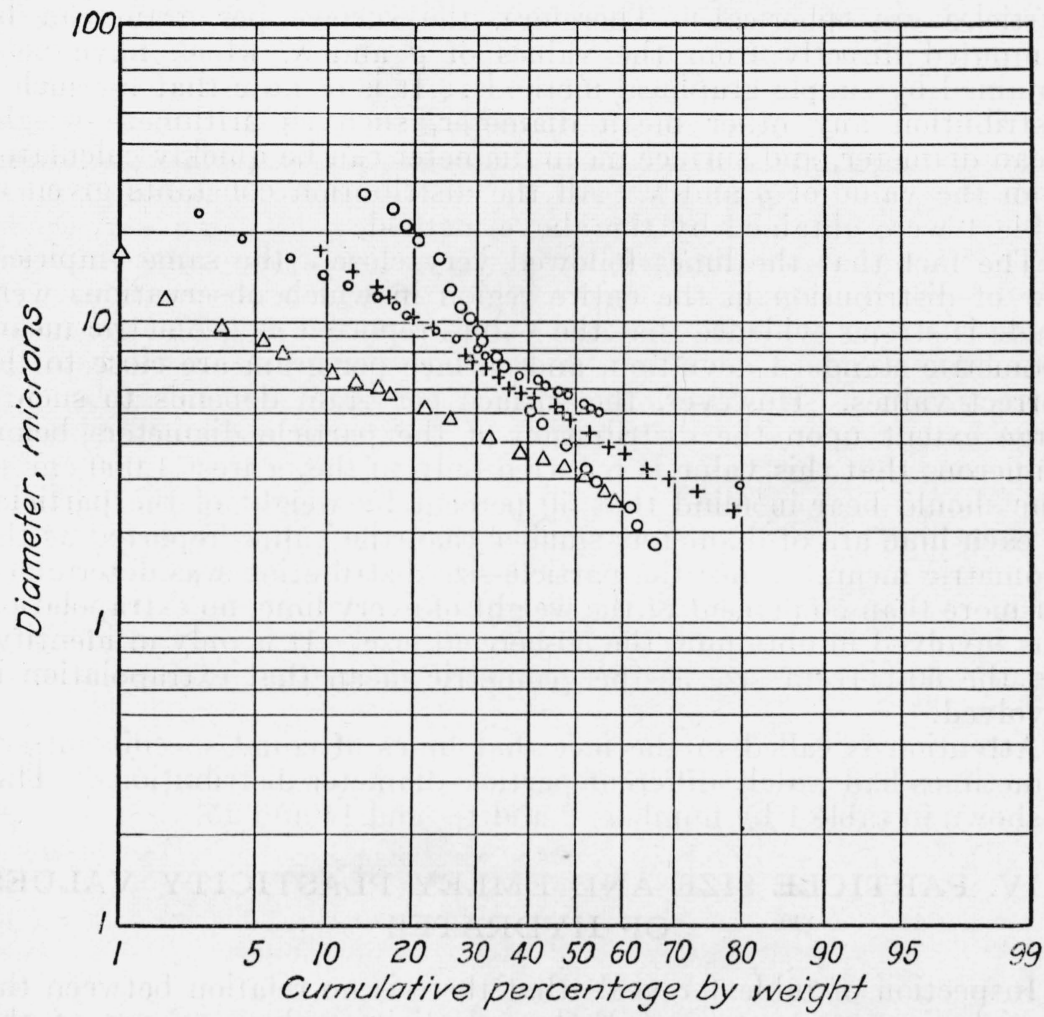

FigURE 1.-Particle-size distribution of four hydrated limes, showing straight line characteristics.

obtained graphically and have pointed out that these two constants completely describe the distribution. For such a distribution the geometric weight-mean diameter, $g$, is that diameter which is exceeded by particles representing half the total weight of material. On a chart such as figure 1 this mean diameter can be read from the ordinate, and corresponds to the position at which a straight line through the plotted points crosses the vertical line representing a cumulative percentage of 50. (The straight lines were fitted by inspection.) The standard geometric deviation, $\lambda$, is a measure of the range of the particle sizes and for such a distribution is the ratio of the diameter of the 50-percent size to the diameter of the 16-percent size.

\footnotetext{
${ }^{5}$ The geometric-weight mean diameter, $g$, is by definition: antilog $\frac{\Sigma w_{d} \log d}{\Sigma w_{d}}$, where $w_{d}$ is the weight of material of diameter $d$. The geometric standard deviation, $\lambda$, is by definition: antilog $\sqrt{\frac{\Sigma w_{d}(\log d-\log g)^{2}}{\Sigma w_{d}}}$.
} 
It can be shown that for such a straight-line distribution the specific surface is given by the formula

$$
s=6 \cdot \frac{\lambda_{2}^{\frac{1}{2} \log _{\mathrm{e}} \lambda}}{\rho g}
$$

where $\rho$ is the density of the material. (It is assumed that the particles are spherical.) Therefore, the surface per gram can be computed directly from the values of $g$ and $\lambda$, which have been obtained by simple graphical methods. It is of note that for such a distribution any other mean diameter, such as arithmetic-weight mean diameter, and surface mean diameter can be quickly calculated from the value of $g$ and $\lambda$. All the distribution constants given in table 1 were obtained by the above method.

The fact that the limes followed very closely the same empirical law of distribution in the entire region in which observations were made is strong evidence that the values reported as geometric mean, geometric standard deviation, and surface per gram are close to the correct values. However, the surface per gram depends to such a large extent upon the distribution of the particle diameters below 2 microns that this value is reported only to the nearest $1,000 \mathrm{~cm}^{2} / \mathrm{g}$. One should bear in mind that 50 percent by weight of the particles in each lime are of diameters smaller than the values reported as the geometric mean. Since the particle-size distribution was determined for more than 50 percent of the weight of every lime, no extrapolation was involved in obtaining the 50-percent size. It is only in identifying the 50-percent size as the geometric mean that extrapolation is involved.

Attention is called to the fact that limes of equal specific surface sometimes had widely different particle-diameter distributions. This is shown in table 1 by numbers 2 and 18 , and 14 and 25 .

\section{PARTICLE SIZE AND EMLEY PLASTICITY VALUES OF HYDRATES}

Inspection of table 1 reveals that there is no relation between the particle-size constants and Emley plasticity values. Some of the nonplastic limes (limes having Emley plasticity values less than 200) are comparatively coarse and others are fine. Many of the plastic hydrates are coarse; but it is not likely that this is the determining factor, for the most plastic hydrate is likewise the finest of all. The distribution curves for two plastic and two nonplastic limes whose particle-size distributions are very similar are shown in figure 2. Not only are all four of these limes very similar but they may be considered as pairs made of a plastic and a nonplastic lime having almost identical distribution characteristics. Figure 3 shows the geometric weight-mean diameters, surface per gram, and Emley plasticity values of the hydrated limes. It is apparent from this graph that there is no correlation between Emley plasticity values and the geometric means or between such plasticity and specific surface. Even though table 1 and figures 2 and 3 do not indicate a relation between particle-size and Emley plasticity values, the fact that distributions below 2 microns were not actually measured leaves the possibility that the distribution of the material finer than 2 microns may be the determining factor in plasticity of limes. 


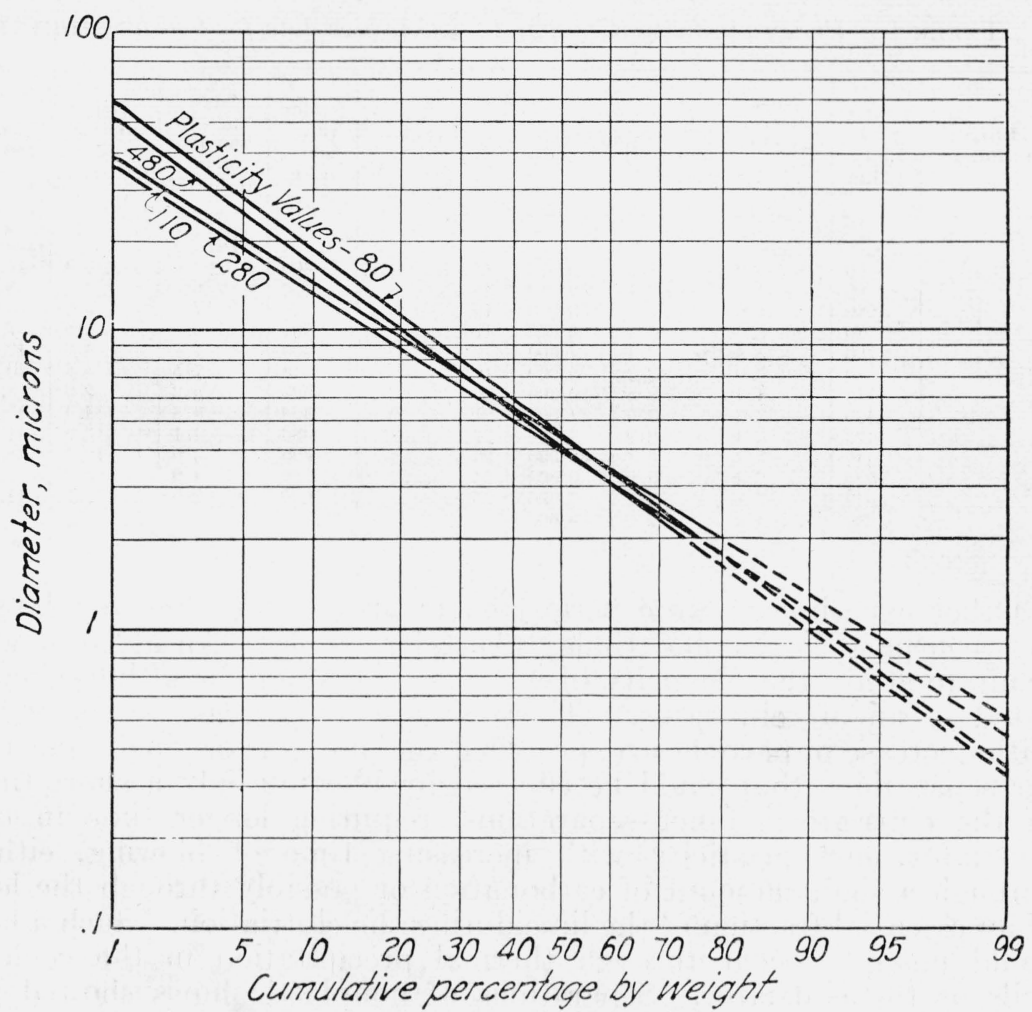

Figure 2.-Particle-size distributions of four hydrated limes having widely varying Emley plasticity values but similar distribution.
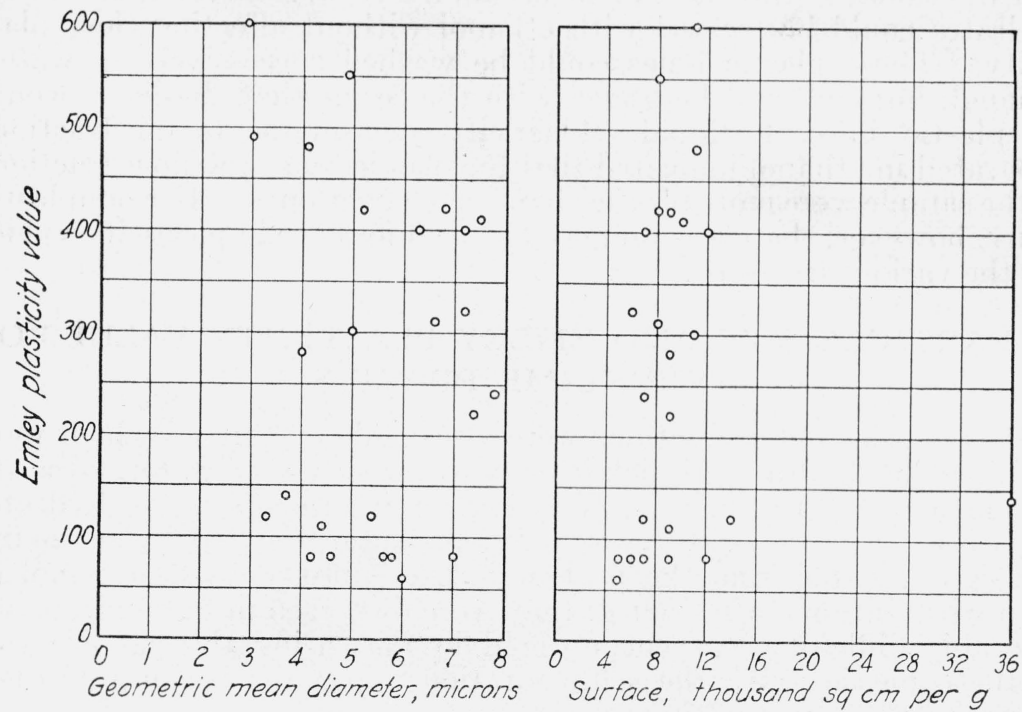

Figure 3.-Emley plasticity values of hydrated limes plotted against their geometric mean diameters and surfaces. 
TABLE 1.-Emley plasticity values and particle-size data for hydrated limes

\begin{tabular}{|c|c|c|c|c|c|c|c|c|c|}
\hline $\begin{array}{c}\text { Lime } \\
\text { number }\end{array}$ & $\begin{array}{c}\text { Emley } \\
\text { plas- } \\
\text { ticity } \\
\text { value }\end{array}$ & $\begin{array}{c}\text { Geo- } \\
\text { metric } \\
\text { weight- } \\
\text { mean } \\
\text { diameter }\end{array}$ & $\begin{array}{c}\text { Geo- } \\
\text { metric } \\
\text { standard } \\
\text { deviation }\end{array}$ & $\begin{array}{l}\text { Specific } \\
\text { surface }\end{array}$ & $\begin{array}{c}\text { Lime } \\
\text { number }\end{array}$ & $\begin{array}{c}\text { Emley } \\
\text { plas- } \\
\text { ticity } \\
\text { value }\end{array}$ & $\begin{array}{c}\text { Geo- } \\
\text { metric } \\
\text { weight- } \\
\text { mean } \\
\text { diameter }\end{array}$ & $\begin{array}{c}\text { Geo- } \\
\text { metric } \\
\text { standard } \\
\text { deviation }\end{array}$ & $\begin{array}{l}\text { Specific } \\
\text { surface }\end{array}$ \\
\hline $\begin{array}{l}1 \\
2 \\
3 \\
4 \\
5 \\
6 \\
8 \\
9 \\
10 \\
11 \\
13\end{array}$ & $\begin{array}{r}60 \\
80 \\
80 \\
80 \\
80 \\
80 \\
110 \\
120 \\
120 \\
140 \\
220 \\
240 \\
280\end{array}$ & $\begin{array}{r}\text { Microns } \\
6.0 \\
4.2 \\
4.6 \\
5.6 \\
5.8 \\
7.0 \\
4.4 \\
3.3 \\
5.4 \\
3.7 \\
7.4 \\
7.8 \\
4.0\end{array}$ & $\begin{array}{l}1.9 \\
3.1 \\
2.7 \\
2.2 \\
2.4 \\
2.2 \\
2.5 \\
3.0 \\
2.2 \\
6.1 \\
3.9 \\
3.4 \\
2.5\end{array}$ & $\begin{array}{r}c m^{2} / g \\
5,000 \\
12,000 \\
9,000 \\
6,000 \\
7,000 \\
5,000 \\
9,000 \\
14,000 \\
7,000 \\
36,000 \\
9,000 \\
7,000 \\
9,000\end{array}$ & $\begin{array}{l}14 \\
15 \\
16 \\
18 \\
19 \\
21 \\
22 \\
23 \\
25\end{array}$ & $\begin{array}{l}300 \\
310 \\
320 \\
400 \\
400 \\
410 \\
420 \\
420 \\
480 \\
490 \\
550 \\
600\end{array}$ & $\begin{array}{r}\text { Microns } \\
5.0 \\
6.6 \\
7.2 \\
6.3 \\
7.2 \\
7.5 \\
5.2 \\
6.8 \\
4.1 \\
3.0 \\
4.9 \\
2.9\end{array}$ & $\begin{array}{r}3.4 \\
3.6 \\
2.8 \\
2.8 \\
4.9 \\
4.5 \\
2.9 \\
4.4 \\
3.0 \\
10.5 \\
2.6 \\
2.1\end{array}$ & $\begin{array}{r}c m^{2} / g \\
11,000 \\
8,000 \\
6,000 \\
7,000 \\
12,000 \\
10,000 \\
8,000 \\
10,000 \\
11,000 \\
110,000 \\
8,000 \\
11,000\end{array}$ \\
\hline
\end{tabular}

Other experiments were performed in an effort to find a relation between particle size and Emley plasticity values. An attempt was made to study the plasticity of various sized fractions of limes separated in an air elutriator. ${ }^{6}$ There was some increase in plasticity with decrease in particle size, provided the lime was originally plastic, for separations that could be effected by blowing only a short time in the elutriator. Finer separations, requiring longer time in the elutriator, lost plasticity with increasing time of blowing, either through a slight amount of carbonation or possibly through the loss of an essential fraction of the lime during the elutriation. Such a loss could possibly occur through thermal precipitation in the cooling coils of the elutriator. Separations of nonplastic limes showed no change in Emley plasticity values for the various sized fractions.

A more successful method of making separations of the limes was the fractional sedimentation in ethanol. It was found that plastic hydrates could be treated with ethanol without affecting their plasticity. These plastic limes could be washed successively in water, ethanol, butanol, and kerosene, with the result that the limes would be plastic in each liquid. Plasticity measurements on fractions separated in ethanol indicated that for plastic limes the finer fractions of the sample were more plastic than the coarser ones. For nonplastic limes, however, there was no measurable difference in plasticity values for the various fractions.

\section{PARTICLE SIZE AND EMLEY PLASTICITY VALUES OF QUICKLIME PUTTIES}

Particle-size determinations were also made on putties which were obtained by hydrating quicklimes with an excess of water, so as to obtain a wet putty without forming an intermediate dry hydrate. Samples for particle-size determinations were prepared by removing the excess water from the putties with ethanol, and the ethanol in turn with butanol, after which they were dispersed in butanol. Considerable difficulty was encountered in measuring the particle size of these putties with the sedimentation apparatus because of their

6 J. A. Swenson, Lacey A.Wagner, George L. Pigman, Effect of granulometric composition of cement on the properties of pastes, mortars, and concrete, J. Research NBS 14, 419 (1935) RP777. 
extreme fineness. Their sedimentation rates were so slow that even at small depths it was impossible to get complete sedimentation in a reasonable time. The data for these quicklimes are, therefore, presented only as a graph (fig. 4) because of the uncertainty in calculating distribution constants where such a large percentage of the material finer than 1 micron would have to be calculated from extrapolations. Even though the quicklime putties were very fine, they

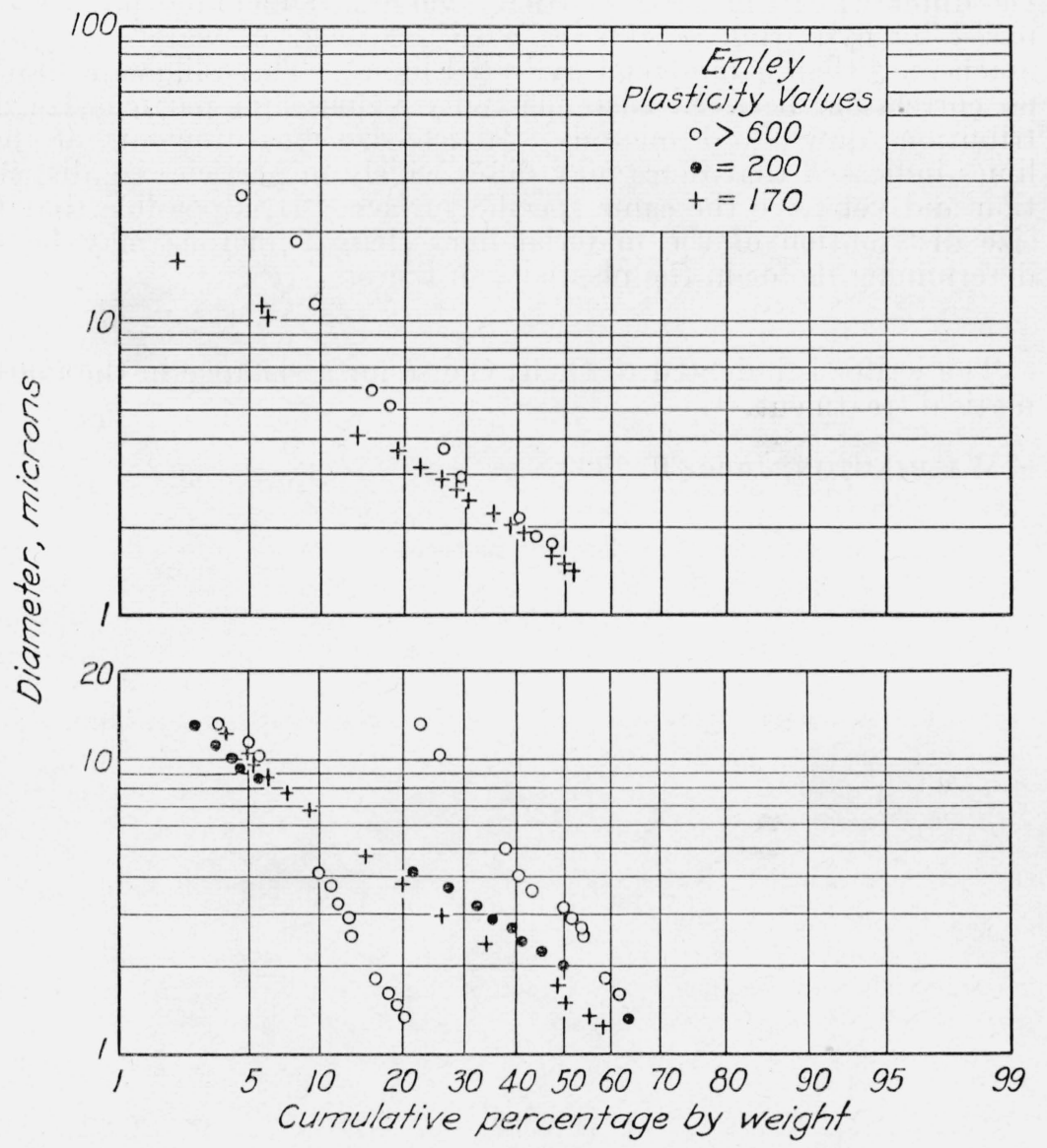

FIgURE 4.-Particle-size distribution of quicklime putties.

showed wide variations in particle size. Some contained considerable quantities of comparatively coarse particles, but all of them had a large percentage of material finer than 1 micron. Quicklimes, when hydrated with an excess of water, yield products which are very much finer than commercial dry hydrates.

The quicklime putties had Emley plasticity values such that some would be classed as nonplastic and some as very plastic limes. Here again the data (fig. 4) for the quicklimes indicate that there is no definite relation between Emley plasticity values and particle size, at least down to 1 micron. 


\section{SUMMARY}

Measurements of the distribution of particle size down to 1 or 2 microns and of Emley plasticity values were made on commercial hydrated limes and on quicklime putties. There was no correlation between Emley plasticity values and the particle-size distributions, at least for the material coarser than 2 microns. Hydrated limes may have very similar particle-size distributions down to 2 microns and yet differ widely in their plasticity values. Quicklime putties prepared by hydrating quicklimes with an excess of water were very much finer than commercial hydrated limes. These likewise showed no correlation between such plasticity values and particle-size distributions down to 1 micron. Particle-size measurements of these limes indicated that limes may differ widely in particle-size distribution and yet have the same specific surface. It is possible that the size distribution of the material finer than 2 microns may be the determining factor in the plasticity of limes.

The writer is indebted to G. R. Gause for assistance in the mathematical treatment.

Washington, June 20, 1939. 